\title{
Clinical evaluation of a rapid, pupil-based assessment of retinal damage associated with glaucoma
}

\author{
Nicholas Wride' \\ Majed Habib' \\ Keith Morris ${ }^{2}$ \\ Steve Campbell ${ }^{3}$ \\ Scott Fraser'
}

'Sunderland Eye Infirmary, City Hospitals Sunderland NHS Foundation Trust, Sunderland, Tyne and Wear, UK; ${ }^{2}$ Applied Neurodiagnostics Ltd, Cramlington, Northumberland, UK; ${ }^{3} \mathrm{School}$ of Health, University of $\mathrm{New}$ England, New South Wales, Australia
Correspondence: Scott Fraser Sunderland Eye Infirmary, Queen Alexandra Road, Sunderland, Tyne and Wear, SR2 9HP, UK $\mathrm{Tel}+44$ I9I 5656256

$\mathrm{Fax}+441915140220$

Email sfraser100@gmail.com
Aims: To evaluate the effectiveness of a new test, the Pupilmetrix ${ }^{\mathrm{TM}}$ PLR60, which uses the pupillary light reflex (PLR) to detect asymmetric retinal damage in patients diagnosed with glaucoma.

Methods: 30 patients, clinically diagnosed as having glaucoma, were recruited to the study, 29 of whom completed testing using the PLR60. A control group of 30 patients who had glaucoma excluded by clinical examination were also recruited and tested using the same protocol on the PLR60.

Results: Of the 110 eyes with test outcomes, overall agreement between the PLR60 result and clinical diagnosis (glaucoma positive or negative) per eye was $84.7 \%$. Sensitivity was $93.1 \%$ (95\% CI 77.2\%-99.2\%) and specificity was 76.7\% (95\% CI 57.7\%-90.1\%). Average (SD) test times (min:sec) for both eyes were 3:21 (0:33) minutes for the glaucoma group and 2:40 (0:35) minutes for the non-glaucoma group.

Conclusions: The results of this preliminary study suggest that the PLR as used in the Pupilmetrix ${ }^{\mathrm{TM}}$ PLR60 test is able to discriminate between patients with glaucomatous retinal defects and those with clinically normal retinas with a diagnostic accuracy that is potentially useful for screening for glaucoma. Test times were markedly quicker than with standard visual field testing.

Keywords: glaucoma, pupillary light reflex, retina, Pupilmetrix

\section{Introduction}

Glaucoma is a progressive optic neuropathy which, if untreated, can lead to severe damage to the visual field. The major risk factor for the disease is intraocular pressure (IOP) which, through an ill-understood mechanism, damages the ganglion cell layer of the retina. This manifests itself as progressive damage to the visual field. The disease is diagnosed by clinical examination of the optic nerve head and the visual field, although because of the high variability involved in visual field testing it can be difficult to identify people with early disease. For this reason there has been a continuing search for a method of objectively examining the retina for signs of glaucomatous damage. The early results of one such method are presented in this paper.

As glaucoma progresses, damage to the retinal nerve fibres is often asymmetric between the upper and lower retina. ${ }^{1,2,3}$ Using these principles, Asman and Heijl ${ }^{4}$ reported the development of the 'Glaucoma Hemifield Test' which used standard perimetric results obtained from the Humphrey field analyser to empirically determine so called 'up-down' differences in the probability maps to detect localized visual field loss. The method is based on the knowledge that early visual field defects (in glaucoma) are frequently restricted to either the upper or lower hemifield and that localized defects are manifested by asymmetries in the differential light sensitivities across the horizontal meridian.

Chen ${ }^{5}$ proposed that this asymmetric change, characteristic of glaucomatous retinal nerve fibre damage, may be detectable as asymmetries in relative sensitivity of the 
pupillary light reflex (PLR) in the upper and lower retinal hemifield. In the paper, Chen also reported the development of a rapid, objective, quantitative measure of asymmetry of this retinal sensitivity, using infrared pupillometry and pairs of large, functionally designed stimuli, symmetrical about the horizontal meridian of the retina.

This approach to determination of retinal asymmetric function within an eye was proposed to be applicable to testing patients with glaucoma. In 2008, Chen ${ }^{6}$ further reported that pupillary evaluation of retinal asymmetry in a test group of 40 glaucoma patients and 40 control patients agreed with perimetry in $70 \%$ of eyes tested.

This current paper reports the first clinical results obtained from a medical device (the Pupilmetrix ${ }^{\text {TM }}$ PLR60; Applied Neurodiagnostics Ltd, Cramlington, UK) designed to employ the test developed by Chen et al ${ }^{5,6}$ to rapidly identify patients with and without glaucoma.

\section{Materials and methods}

The study was wholly conducted at Sunderland Eye Infirmary, Sunderland, UK and was approved by the UK NHS National Research Ethics Service (NRES) and the local R and D committee. Participants were patients attending the hospital and two groups were identified - one with clinical diagnosis of glaucoma and a second who clinically did not have glaucoma or personal history or family history of the disease. Both sets of patients were examined by the same person (an ophthalmologist with a special interest in glaucoma) and all underwent the same tests for glaucoma (ie, IOP measurement, disc examination, visual field testing). Control patients were taken from other clinics in the hospital. Subjects with known optic neuropathies, eg, ischemic and other eye conditions such as retinal detachment that could potentially show retinal asymmetry, were excluded from both groups. Patients who were either not deemed able to sit comfortably at the equipment or whose vision was too poor to maintain fixation were excluded. All test subjects gave their informed consent to participate in the study.

The Pupilmetrix TM PLR60 (Figure 1) carries out a test of retinal function. It combines the established and well understood PLR with infrared pupilometry (IRP) technology to provide data on the response of the pupil to a series of specially designed stimuli. Both PLR and IRP are commonly used in ophthalmology and optometry. Using specially designed software the device calculates a result that indicates the presence or absence of asymmetric damage to the nerve fibre layer of the retina.
Examination takes place in a darkened room with minimal distractions (noise and light). After a short period of dark adjustment, the subject is asked to lean on the chin and head rest and look at a red-cross (fixation target) that appears on the stimulus screen. The non-test eye is covered with an eye patch and the pupilometer is focused on the test eye.

Each eye is tested independently by stimulating the retina with a controlled and defined sequence of three different shapes (stimuli). The shapes are mirror images about a horizontal meridian that also artificially divides the retina into an upper and lower half. During the stimulus sequence the shape halves vary in brightness relative to each other and their background. The shapes alternate in brightness at a frequency of 1 per second for 2 seconds per stimulus pair.

Three stimulus pairs constitute a stimulus cycle of 6 seconds. Three cycles represent one scan. The stimulus shape then changes and the regime repeated to complete the second scan. The stimulus shape changes to the third one and the regime is repeated. Each whole 'scan' therefore lasts 18 seconds and the subject can relax between scans.

The pupilometer comprises a source of near infrared light that illuminates the pupil to form a 'bright pupil' against a dark background of the iris and a video camera that records images of the illuminated pupil at a frequency of $60 \mathrm{~Hz}$. Eye tracking software superimposes a circle that defines the circumference of the illuminated pupil. This is called 'pupil discrimination' and is the basis of tracking changes in the pupil diameter.

As the upper and lower stimuli change in brightness relative to their background, the pupil dilates or contracts in response. This is the pupillary light reflex. The pupilometer detects and records these changes.

\section{Results}

\section{Patient details}

Thirty non-glaucoma patients and 30 glaucoma patients were tested. One glaucoma patient was excluded from the study because of inability to sit comfortably at the PLR60, discovered after the test was begun. Patients were not age or sex matched due to the consecutive nature of the recruitment.

The mean (SD) and range of age of the non-glaucoma group was 59.6 (16.7), range 20 to 82 years and 69.9 (13.6), for the glaucoma group mean (SD), range 24 to 91 years.

A total of 118 eyes were tested. In all patients a test outcome was obtained in at least one eye but could not be obtained for 8 eyes $(6.8 \%)$. In 5 of these cases, this was due to the patient blinking 'excessively' (greater than 9 times) so preventing enough pupil diameter data to enable a contrast 



Figure I The Pupilmetrix TM PLR60. a) Device layout; b) Patient positioning; c) The peripheral stimulus pattern. 
balance result to be calculated. The remaining 3 losses were due to poor data quality preventing the PLR60 software from calculating a result. Poor data quality typically arose when other stimuli, eg, sudden sounds and distractions overwhelmed the intended stimulus resulting in poor correlation between the three stimulus cycles that comprise each scan and preventing meaningful calculation of a result. The possibility of reducing data loss due to these causes is being investigated.

None of the patients tested required correction of their sight in order to adequately see the fixation target and stimuli.

\section{Sensitivity and specificity by patient}

Results were analyzed for sensitivity and specificity using the clinical diagnosis as the reference standard. Since the intended use of the PLR60 is for the detection of (screening for) glaucoma, sensitivity and specificity were estimated per patient (rather than per eye) recording retinal damage in either eye as a test positive result.

The receiver operator curve (ROC), was calculated using the highest absolute value of the six contrast balance values for each patient and is shown in Figure 2. Area under the curve was 0.907 with a standard error of 0.0408 and $95 \%$ confidence interval (CI) of 0.802 to 0.967 .

Using a $2 \times 2$ diagnostic table, sensitivity and specificity were calculated to be $93.1 \%$ (95\% CI 77.2\%-99.2\%) and $76.7 \%$ (95\% CI 57.7\%-90.1\%).

In both cases, 'no test outcomes' were excluded from the analysis.

\section{Agreement with clinical diagnosis}

The level of agreement between the PLR60 test outcome and clinical diagnosis was estimated for each eye tested

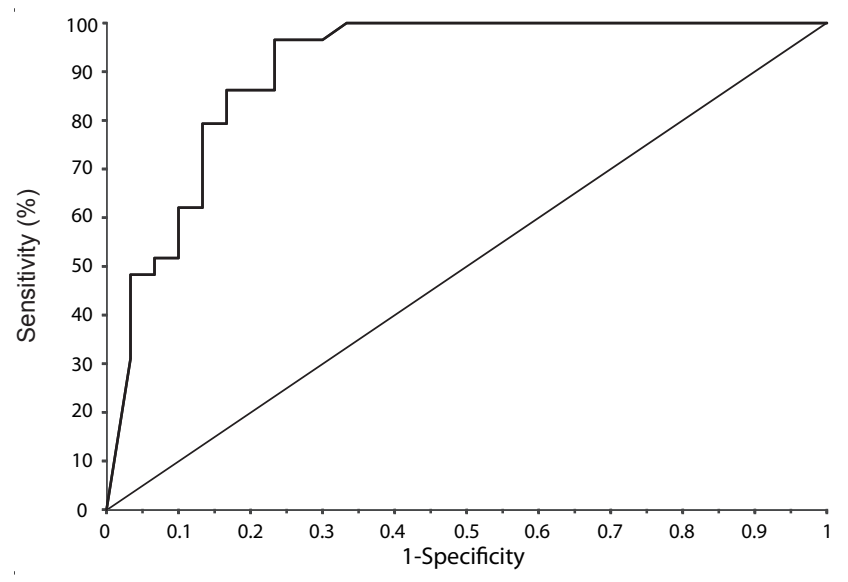

Figure 2 Receiver operator curve. and statistical significance determined using kappa values. Overall agreement between the PLR60 test outcome and clinical diagnosis (glaucoma positive or negative) was $84.7 \%$ $(\mathrm{K}=0.6957,95 \% \mathrm{CI}, 0.5153-0.8761)$.

Of the 110 eyes with test outcomes, 53 were from glaucoma patients and 57 were from control subjects. Of the 29 glaucoma patients, 9 had bilateral glaucoma and the remainder had glaucoma in one eye only. Agreement between the PLR60 test outcome and clinical diagnosis (glaucoma positive or negative) per eye for the glaucoma positive group was 43/53 (81\%). For the control group, agreement between the PLR60 test outcome and clinical diagnosis (glaucoma negative) per eye was 50/57 (87.7\%).

\section{Test time}

Test times were calculated from the number of scans taken to obtain a result for each eye multiplied by the fixed time for each scan and so represent the time the PLR60 took to scan both eyes not the overall duration of the test. Average (SD) test times (min:sec) for both eyes were 3:21 (0:33) minutes for the glaucoma group and 2:40 (0:35) minutes for the non-glaucoma group. Figure 3 shows the distribution of test times. The control group took less time to complete the test, with $90 \%$ of patients completing the test for both eyes in 3.5 minutes and all completing within 4 minutes. For the glaucoma group, the figures were 4 minutes and 4.5 minutes respectively.

\section{Discussion}

A detailed description of the principles of the Pupilmetrix ${ }^{\mathrm{TM}}$ PLR60 test can be found in Chen. ${ }^{5}$ In summary, the relative pupillomotor sensitivity is determined for corresponding superior and inferior retinal territories within the same eye. The test also uses stimuli larger than those used in classic perimetry to elicit the PLR response. These stimuli shapes are named the Paracentral, Bjerrum and Peripheral (out to 20 degrees) and cover a large portion of the area of the visual field most subject to glaucomatous loss. ${ }^{1}$ This may be thought of as using the same retina as a control and an internal control of this sort could potentially reduce test-retest variability.

During the test, the non-test eye is covered to exclude light. The patient observes the stimuli on an LCD screen and fixes their gaze on a small target cross. There is no direct interaction between the patient and the device since an inbuilt infra-red pupilometer automatically tracks the changes in pupil diameter in response to the stimuli. This feature of the test provides the objectivity that is sought after in tests of visual field function. 


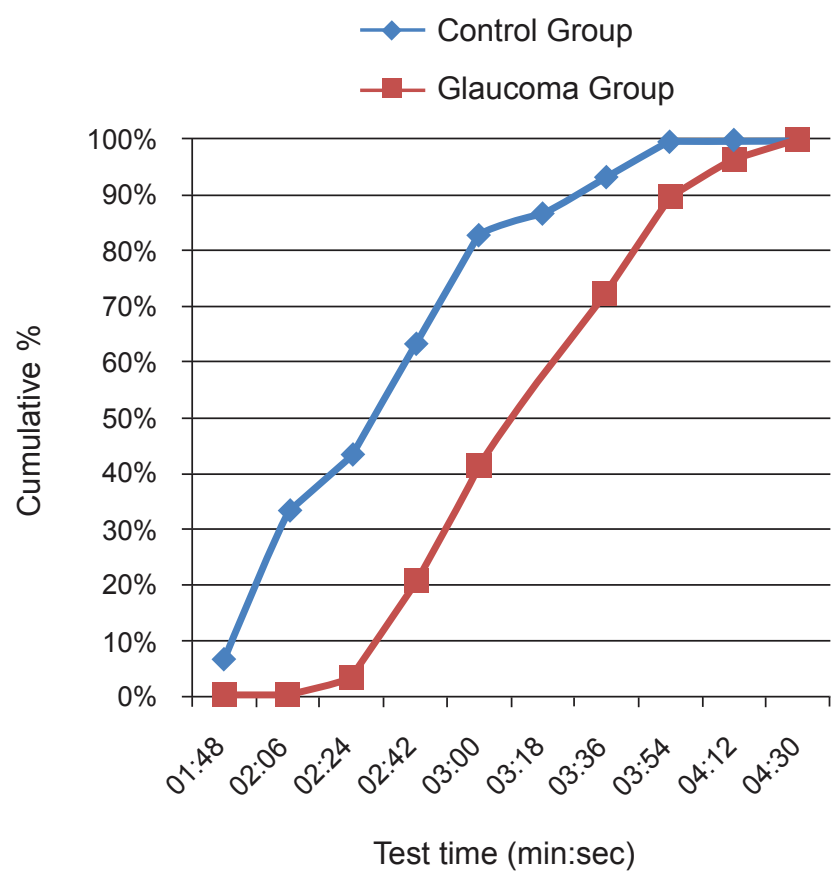

Figure 3 Distribution of test times.

An effect of the large sizes of the stimuli used in the PLR60 test is that correction is not required within \pm 6 dipotres of focus on the fixation target (HJ Wyatt, personal communication). At the distance between the subject's eye and the stimulus screen this means that patients who are between $+1.5 \mathrm{D}$ hyperopic (in the absence of accommodation) and $-10.5 \mathrm{D}$ myopic will be satisfactorily focused on the stimuli.

The test yields a contrast balance (CBal) value for each stimulus shape indicating the relative sensitivity to the upper and lower stimuli and thus the level of asymmetric damage present in each region of the stimulated retina. The closer the magnitude of the Cbal result is to zero the less asymmetric damage is present. Numerical CBal values for each stimulus pair (scan) are categorized as normal, marginal or abnormal by comparing the result for each stimulus shape to preset limit values. The test outcome summarizes the results of each set of scans for each eye and shows either no damage is indicated where all three scans are within normal limits, damage is suspected where at least one scan shows a 'marginal' defect, or damage is indicated where at least one scan shows an abnormal defect. Damage suspected and damage indicated results were treated as 'test postives'. No damage indicated was treated as 'test negative'.

In their Health Technology Assessment Report of 2007 for The National Institute for Health Research, Burr et al undertook a systematic review of studies that reported the diagnostic accuracy of various screening tests for open angle glaucoma. ${ }^{7}$ The sensitivity and specificity results for the PLR60 compare well with those of other tests of visual function and eye examination reported by Burr. For example, standard automated suprathreshold perimetry was shown to have a sensitivity of $71 \%$ and a specificity of $85 \%$ by determining summary receiver-operating characteristics for these parameters from 9 studies. $^{8}$ The sensitivity of the PLR60 test is high at $93.1 \%$ compared with other tests studied in the assessment. The specificity of $76.7 \%$ may be considered somewhat low for a screening test but again is comparable to that of other tests studied. A possible hypothesis could be that the PLR60 test is detecting early stage (ie, earlier than can be diagnosed clinically) retinal damage associated with glaucoma (or possibly other sub-clinical optic neuropathies) although this needs to be investigated. In the context of optometric screening (usually every 2 years in the UK), the specificity of the PLR60 is unlikely to be a problem, the high sensitivity being important to detect glaucoma as and when it occurs. It should also be noted that the absence of age matching could potentially artificially increase the diagnostic accuracy of the test.

One glaucoma patient, diagnosed with advanced field losses in both eyes, was not detected as having retinal asymmetric damage in either eye. The visual field data showed extensive loss in both hemispheres of both eyes, so much so that there was no detectable evidence of asymmetric damage to the retina. The PLR60 detects differences in the degree of damage to the upper and lower retina and where there is no difference will indicate no damage detected. Again, in the context of optometric screening, it is unlikely for a patient to present with such advanced damage and if this were to occur, the other tests carried out as part of eye examination should detect the disease. Should the Pupilmetrix ${ }^{\mathrm{TM}}$ test be used in the clinical setting in isolation, this limitation would be more important but again would be mitigated to a large extent by the results of other tests and examinations carried out during diagnosis.

The PLR60 test times, for both glaucoma and control patients were in the order of 4 minutes for both eyes. Slightly longer test times were seen with glaucoma patients, due to the PLR60 repeating scans that indicate an abnormality in the retinal response in order to gain a more reliable test outcome. These test times are comparable to the 5 minutes median times reported by Spry ${ }^{9}$ for both standard automated perimetry and frequency doubling technology and the 3- to 6-minute test times using Henson screeners reported by Tuck and Crick. ${ }^{10}$ It is recognized that the total time to test a patient, which includes preparation and explanation 
times will be longer than the test times reported here. To be consistent with many manufacturers of field test equipment the machine test time and not the overall time is reported. Tuck and Crick $^{10}$ report the explanation and test times for various modes of field screening tests and show them to be in the order of a ratio of $1: 2$, ie, the explanation time is about half of the test time.

In this study, the PLR60 showed high levels of agreement (over 80\%) with the clinical diagnosis of glaucoma both per patient and per eye. The incidence of no test outcomes being returned for one or other eye $(6.8 \%)$ is considered to be low and suggests the generally good tolerance patients had to the test. The objective nature of the PLR60 test, namely that the patient does not have to make any decisions about the stimulus, is considered to be a significant factor in achieving good patient tolerance and anecdotal feedback from patients supported this. It is expected that in regular use such instances would be managed and reduced by re-testing the eye after resting the patient and/or administering eye drops (eg, artificial tears if the patient had dry eyes) if appropriate.

The PLR60 device is a test of visual function and is intended for use in Optometric practice as part of the routine eye examination. The relatively high sensitivity and specificity and quick test times achieved in this study suggest that this is feasible. The performance of current screening methods is generally considered poor, with the specificity of referrals by optometrist being quoted in the range of $40 \%$ to $50 \% .^{11,12}$ With field testing, patient satisfaction is generally low and the time taken has potential economic impacts for the optometrists.

Whilst this study involved patients that were likely to perform well on the PLR60, it does suggest that the Pupilmetrix ${ }^{\mathrm{TM}}$ PLR60 test has features (objectivity, high sensitivity and specificity, speed and patient comfort) that can benefit both the patient and practitioner.

Additional studies with larger numbers and consecutive patients will help to more fully elucidate the role of this test in everyday practice and determine the test's sensitivity and specificity when compared to the clinical diagnosis of multiple clinicians.

\section{Acknowledgments}

The authors are grateful to Professor Harry J Wyatt, Emeritus Professor, Biological Sciences, State College of Optometry, State University of New York, USA for his advice and guidance during the study and Dr Thomas Keller of Acomed Statistik, Leipzig, Germany for the statistical analysis of the results.

The study was sponsored by City Hospitals Sunderland Foundation NHS Trust and funded through a grant from Applied Neurodiagnostics Ltd.

\section{Disclosures}

KM is employed by Applied Neurodiagnostics Ltd, UK. None of the other authors have any conflicts of interest to disclose.

\section{References}

1. Aulhorn E, Karmeyer H. Frequency distribution in early glaucomatous visual field defects. Documenta Ophthalmologica. 1977;14:75-83.

2. Hart WM Jr, Becker B. The onset and evolution of glaucomatous visual field defects. Ophthalmology. 1982;89(3):268-279.

3. Åsman P, Hejl A. Evaluation of methods for automated hemifield analysis in perimetry. Arch Ophthalmol. 1992;110(6):820-826.

4. Åsman P, Heijl A. Glaucoma Hemifield Test. Automated visual field evaluation. Arch Ophthalmol. 1992;110(6):812-819.

5. Chen Y, Wyatt HJ, Swanson WH. Pupillary evaluation of retinal asymmetry: development and initial testing of a technique. Vision Res. 2005;45(19):2549-2563.

6. Chen Y, Wyatt HJ, Swanson WH, Dul MW. Rapid Pupil-Based Assessment of Glautomatous Damage. Optom Vis Sci. 2008;85(6):471-481.

7. Burr JM, Mowatt G, Hernandez R, et al. The clinical effectiveness and cost-effectiveness of screening for open angle glaucoma: a systematic review and economic evaluation. Health Technol Assess. 2007; 11(41):41-83.

8. Burr JM, Mowatt G, Hernandez R, et al. The clinical effectiveness and cost-effectiveness of screening for open angle glaucoma: a systematic review and economic evaluation. Health Technol Assess. 2007; 11(41):63.

9. Spry PG, Hussin HM, Sparrow JM. Clinical evaluation of frequency doubling technology perimetry using the Humphery Matrix 24-2 threshold strategy. Br J Ophthalmol. 2005;89(8):1031-1035.

10. Tuck MW, Crick RP. Screening for glaucoma: the time taken by primary examiners to conduct visual field tests in practice. Ophthalmic Physiol Opt. 1994;14(4):351-355.

11. Vernon SA, Ghosh G. Do locally agreed guidelines for optometrists concerning the referral of glaucoma suspects influence local practice? Eye. 2001;15:458-463.

12. Tuck MW, Crick RP. Relative effectiveness of different modes of glaucoma screening in optometric practice. Opthalmic Phyisol Opt. 1993;13(3):227-232. 